\title{
O uso de geoprocessamento na análise populacional: determinação do padrão de desenvolvimento dos municípios mineiros a partir da relação entre a frequência escolar e a fecundidade das mulheres nos censos de 1991 e 2000
}

\section{The use of GIS in population analysis: pattern determination of the development of mining towns from the relationship between school attendance and fecundity of women in the censuses of 1991 and 2000}

Ramon Messias Martins

Graduanda em Geografia - Instituto de Geociências - UFMG messias.ramon@gmail.com

Vanessa Marques Viana Graduanda em Geografia - Instituto de Geociências - UFMG vanessamv88@gmail.com

Artigo recebido para revisão do evento em 22/04/2014, aceito para publicação em 11/05/2014 e recebido para publicação em 01/06/2014

\section{Resumo}

O Brasil assim como outros países do mundo tem passado pelo processo de Transição Demográfica, apesar de apresentarem estágios diversos. Os países europeus, por exemplo, já se encontram na fase mais avançada da transição em que o crescimento vegetativo chega a ser negativo devido à queda vertiginosa da fecundidade. No Brasil, pode-se dizer que a transição se encontra na fase de queda da fecundidade, se aproximando da taxa de reposição de 2,1 . No entanto, o território brasileiro é muito expressivo, o que denota diferenças regionais, logo, em alguns estados da região norte a fecundidade está acima de 3,0 filhos por mulher, enquanto nos estados do Sul e Sudeste as taxas estão abaixo de 2,1. Diversos demógrafos acreditam que a fecundidade tem relação direta com o acesso a educação por parte das mulheres. A partir desses levantamentos, o presente trabalho visa cruzar as taxas de fecundidade dos Censos de 1991 e 2000 com a frequência escolar das mulheres em idades entre 15 e 49 do estado de Minas Gerais. O resultados foram espacializados no ArcGis $10 ®$ para fins de comparação do anos estudados. Percebe-se que houve um avanço de 1991 para 2000, pelo fato de um maior número de municípios encaixarem na dinâmica dos países desenvolvidos.

Palavras-chave: Fecundidade; Transição Demográfica; Escolaridade.

\begin{abstract}
Brazil and many countries around the world have gone through a process called Demographic Transition. The European countries are already in an advanced transition stage when the vegetative growth becomes negative due to sharp drop in fertility. In Brazil, we can say that the transition is in the process of fertility decline, approaching the replacement rate of 2.1. However, Brazil is showing huge regional differences, so in some northern states of fertilities above 3.0, while in the South and Southeastern rates are below 2.1. Many demographers believe that fertility is directly related to access to education for women. From these surveys, the objective of the present work aims at cross rates fertility censuses of 1991 and 2000 with the attendance of women aged between 15 and 49 of the state of Minas Gerais. The results were spatialized in ArcGIS 10® for comparison of the years studied. It is felt that there was an improvement from 1991 to 2000, because a larger number of municipalities in the dynamic fit of the developed countries.
\end{abstract}

Keywords: Fertility; Demographic Transition; Education. 


\section{INTRODUÇÃO}

A composição de uma população é o reflexo de sua dinâmica ao longo do tempo e está ligada diretamente aos níveis de Fecundidade, Natalidade e Mortalidade, o que controla o ritmo de crescimento populacional. Toda a população mundial vem passando por um processo de transição demográfica. Alguns países se encontram em fases mais avançadas e outros estão nas fases iniciais.

Para Yazaki (2003) um aspecto que caracteriza as populações em diversos países, principalmente europeus e asiáticos, na atualidade é a insuficiência da fecundidade para a substituição de suas próprias gerações. A Taxa de Fecundidade consiste na capacidade de reprodução de determinada sociedade, e expressa a condição reprodutiva média das mulheres (de 15 a 49 anos) de um determinado local, sendo um dado importante na análise da dinâmica demográfica.

O mínimo indicado para que a população se mantenha é de 2,1 filhos por mulher, o que equivale à taxa de reposição populacional. Segundo a Organização das Nações Unidas (ONU), em seu Relatório sobre a Situação da População Mundial Países de 2011, países desenvolvidos como a Inglaterra, Itália, Japão e Canadá, dentre outros, experimentam um momento em que as taxas de fecundidade estão abaixo de 1,7 filhos por mulher, ou seja, abaixo da taxa de reposição.

Até o século XVII as taxas de fecundidade e de mortalidade mundiais eram altíssimas e retraiam o crescimento da população (Gráfico 1). Esses fatores fazem Landry (1934) definir esse período como um momento de crescimento populacional primitivo.

Gráfico 1 - Evolução do crescimento da população mundial

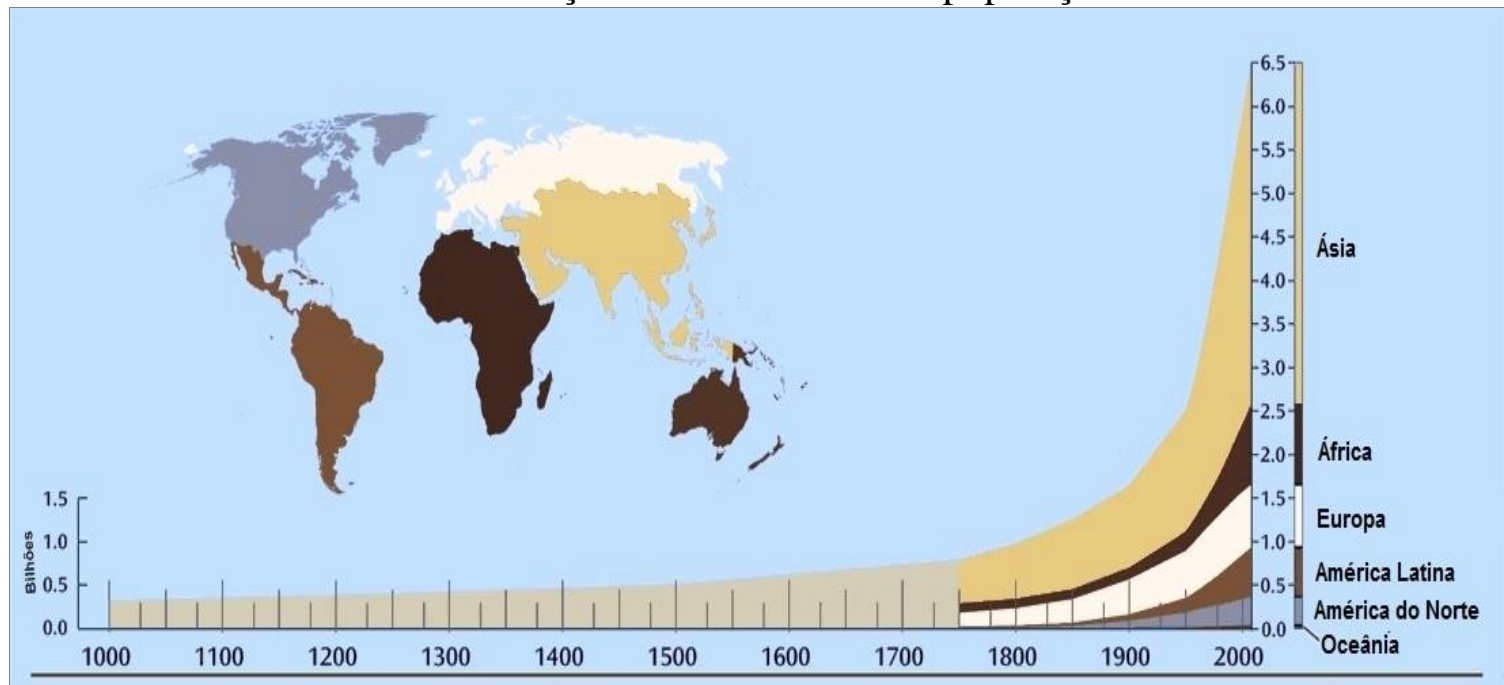

Fonte: Adaptado de 1- Departamento de Assuntos Econômicos e Sociais das Nações Unidas, World Population Prospects: The 2004 Revision and World Prospects: The 2003 Revision, <http://esa.un.org/unpp> 2- Nações Unidas, 1973. "The Determinants and Consequences of Population Trends, Vol.1" "World Population Prospects: The 1998 Revision", <http://www.geohive.com/global/> 
A partir do século XVII ocorre no mundo uma fase de estabilizado crescimento natural e, no século XVIII, ocorre um crescimento considerável da população mundial. Esse aumento é legado do processo de urbanização em que passava o continente europeu durante a revolução industrial. Um crescimento expressivo ocorre no século XIX, entretanto, neste momento os países industrializados já apresentam declínio da fecundidade, da mortalidade e o crescimento natural da população diminui de intensidade configurando a transição demográfica.

Diferentemente, os países em desenvolvimento tiveram sua modificação nos índices populacionais e na transição demográfica, após 1940. Segundo Faria et. al., (2012) tais países foram responsáveis pela explosão demográfica que ocorreu entre os anos 40 e 70. Em apenas 50 anos, o Brasil passou pelo processo que a Europa levou mais de 150 anos para concluir.

Um dos componentes mais importantes da dinâmica de transição demográfica é a Taxa de Fecundidade. O Brasil, por exemplo, configurou mudanças significativas na transição da população no momento em que a Taxa de Fecundidade manteve forte tendência de queda. Na década de 1970 a taxa era de 5,8 filhos por mulher. Em 2000 era de 2,38 e, em 2010 a taxa chega a 1,9, ou seja, abaixo da taxa de reposição de 2,1 filhos por mulher.

Autores como Merrick \& Berquó (1983) e Alves (2002) propõem que esse declínio da fecundidade é o elemento principal da aceleração da transição demográfica. A mudança no comportamento reprodutivo das mulheres residentes em áreas urbanas, o crescimento dos níveis educacionais e diminuição do analfabetismo e o crescimento da taxa de participação feminina no mercado de trabalho são fatores decisivos para a transição.

Dados refletem que a Taxa de Fecundidade no Brasil, próxima de 2 filhos por mulher, na primeira década do século XXI, pode alcançar entre 1,6 e 1,8 filhos após 2020. Valores dos últimos Censos permitem traçar uma tendência de declínio da fecundidade total. A taxa brasileira passou de 6,16, em 1940, para 1,9, entre 2000 e 2010 (Gráfico 2). Perpétuo \& Wong (1999) citados por Alves (2002) verificaram nos anos 2000 taxas abaixo de 2,0 nos grupos socioeconômicos mais favorecidos da população brasileira.

Numa altura em que a população mundial superou a barreira dos 7 bilhões de habitantes (ONU, 2013), baixas taxas de fecundidade são uma preocupação. No Brasil, a redução da fecundidade significa em médio prazo menos pessoas a entrar no mercado de trabalho, uma tendência que põe em risco o crescimento econômico nacional e gera diversas implicações sociais.

Farias et. al., (2012) cita que a inserção da mulher no mercado de trabalho e o aumento do seu nível de escolaridade influenciam diretamente na taxa de fecundidade. Esse argumento é levantado pelos autores tendo em 
vista que em regiões onde a população feminina é mais pobre e com menos informação a taxa de fecundidade não apresenta redução significativa, como é o caso do nordeste brasileiro e do norte de Minas, no Vale do Jequitinhonha e Mucuri.

Gráfico 2 - Taxa de Fecundidade conforme região do país

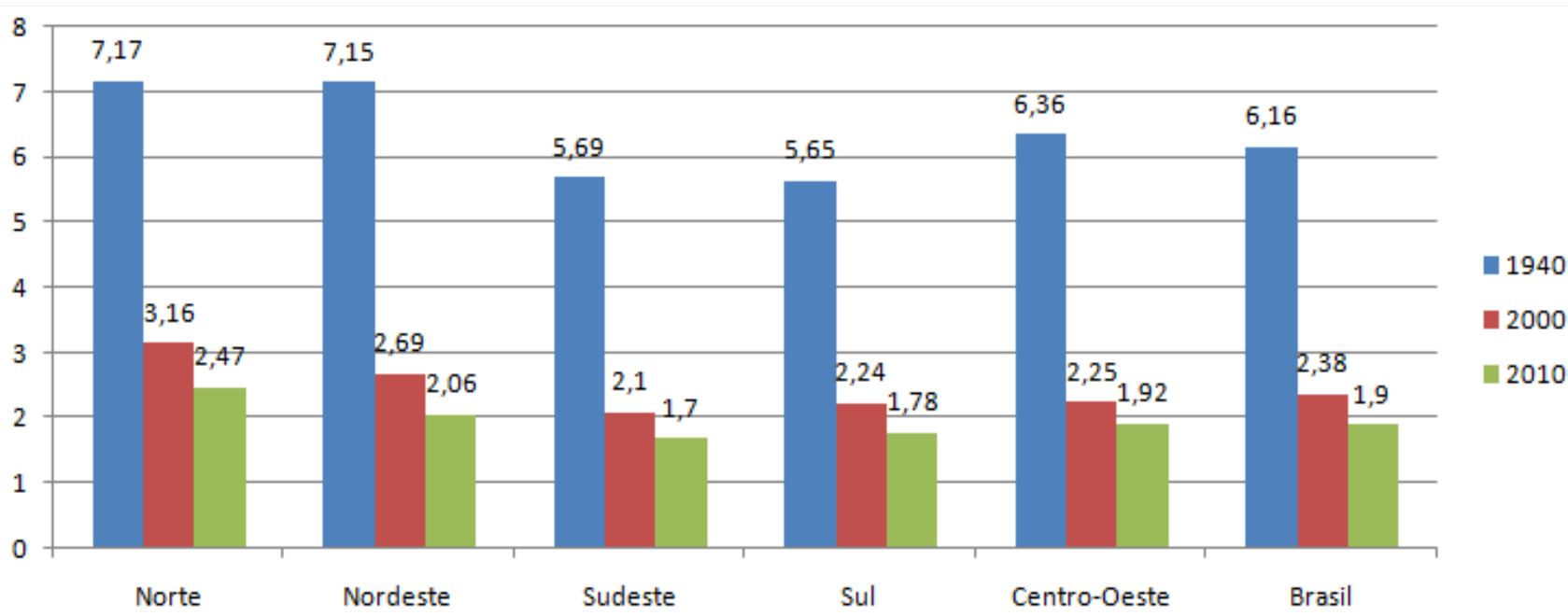

Fonte: Elaborado pelos autores. Base de dados IBGE.

Dados do IPEA (Instituto de Pesquisa Econômica Aplicada) também corroboram com a proposição dos autores op. cit. O relatório da Pesquisa Nacional por Amostra de Domicílios de 2009 evidencia queda da Taxa de Fecundidade relacionada à fatores como o nível de escolaridade. A proporção revela que, em 1992, uma mulher brasileira com nível de educação mais baixo tinha 1,8 filho a mais do que aquelas com escolaridade mais alta. O estudo também mostra, que a Taxa de Fecundidade é mais alta entre as pessoas de baixa renda, embora os diferenciais estejam sendo reduzidos ao longo do tempo. Em 1992, a diferença era de 3,4 filhos entre as mulheres pobres e ricas. Em 2008 a taxa se fixou em 2,2 filhos.

Baseado nos fundamentação levantada, o presente trabalho se justifica devido às relações feitas através do cruzamento de dados de fecundidade e frequência escolar feminina no Ensino Médio, obtendo resultados que sustentam as informações contidas no texto acima e evidencia a evolução do desenvolvimento dos municípios mineiros representados por mapas, com dados do censo de 1991 e 2000.

\section{OBJETIVO}

Para consecução de tal proposta será necessário responder aos seguintes objetivos específicos:

Elaborar mapas para os anos de 1991 e 2000 em que estejam relacionados o nível de escolaridade das mulheres mineiras e a mudança nos níveis de fecundidade; 
Comparar os dados de 1991 e 2000, bem como os mapas desenvolvidos a partir desses, para traçar um panorama do desenvolvimento da fecundidade das mulheres em Minas Gerais na medida em que o nível de escolaridade se eleva.

\section{METODOLOGIA}

Para a elaboração deste trabalho primeiramente foi realizada uma revisão bibliográfica acerca do assunto tratado. Em seguida, foram retirados do site do IBGE os dados relacionados às taxas de fecundidade e à frequência escolar das mulheres entre 15 e 49 anos no Ensino Médio dos anos de 1991 e 2000. Após isso, os dados foram inseridos no Excel 2007 e salvos na extensão DBF. Ainda no site do IBGE, a malha viária municipal de Minas Gerais de 2007 foi disponibilizada.
A partir da shape da malha municipal digital inserida no ArcGis, juntamente com as tabelas no formato DBF,foi possível gerar arquivos MDX para as duas variáveis, fecundidade e escolaridade, trabalhadas para os dois anos selecionados, 1991 e 2000.

Separadamente, cada arquivo foi classificado em quatro classes com escalas fixas para fins de comparação. Foi utilizado o intervalo fixo por este proporcionar a comparação entre as classes, e técnicas de Apoio Multicritério à Decisão (AMD). Seguido, os arquivos foram transformados em raster e classificados em classes numeradas de 1 a 4 , quanto menor é o valor atribuído, melhor a qualidade do atributo. A Taxa de Fecundidade Total e a Frequência Escolar foram classificadas assim:

Tabela 1 - Classes de Fecundidade Total

\begin{tabular}{|l|l|l|}
\hline \multicolumn{3}{|c|}{ TAXA DE FECUNDIDADE TOTAL } \\
\hline Classes & Valores (1991) & Valores (2000) \\
\hline $\mathbf{1}$ & $1,95-2,1$ & $1,65-2,1$ \\
\hline $\mathbf{2}$ & $2,1-3,5$ & $2,1-3,5$ \\
\hline $\mathbf{3}$ & $3,51-5,12$ & $3,51-5,12$ \\
\hline $\mathbf{4}$ & $5,13-6,96$ & $5,13-6,96$ \\
\hline
\end{tabular}

Fonte: Elaborado pelos autores.

Tabela 2 - Classes de Frequência Escolar

\begin{tabular}{|l|l|l|}
\hline \multicolumn{3}{|c|}{ FREQUÊNCIA ESCOLAR DAS MULHERES NO ENSINO MÉDIO } \\
\hline Classes & Valores (1991) & Valores (2000) \\
\hline $\mathbf{1}$ & $50,01-65$ & $50,01-65$ \\
\hline $\mathbf{2}$ & $35,01-50$ & $35,01-50$ \\
\hline $\mathbf{3}$ & $20,01-35$ & $20,01-35$ \\
\hline $\mathbf{4}$ & $0,16-20$ & $6,51-20$ \\
\hline
\end{tabular}

Fonte: Elaborado pelos autores.

Após esse procedimento de pesos de $60 \%$ para a frequência escolar e de reclassificação dos arquivos raster de cada $40 \%$ para a fecundidade segundo referências de variável para cada ano, utilizou-se a ferramenta ADM.

Raster Calculator. Para isso foram atribuídos os 
A definição das classes: valores em diferentes classes de um universo subdesenvolvido, desenvolvimento e amostral e por ser realizado de modo automático desenvolvido foi realizada através da no software ArcGis.

computação da média simples e ponderada da Após a classificação dos municípios em Frequência Escolar e da Taxa de Natalidade. seus respectivos valores de índice, foram Essas médias foram padronizadas para escala 0 gerados mapas temáticos em ambiente do a 1. Os intervalos: a) Desenvolvido sistema de informação geográfica (SIG) que (desenvolvimento muito alto), b) Em permitiu a espacialização dos dados na malha Desenvolvimento (desenvolvimento médio e estadual. Esses mapas são apresentados nos alto) e c) Subdesenvolvido (desenvolvimento resultados e respalda a discussão apresentada baixo) seguiram o método de quebra natural de nesse artigo.

valores. Esse método foi escolhido por ser $\quad$ O fluxograma abaixo sintetiza as etapas conhecido por determinar o melhor arranjo de realizadas para a conclusão deste trabalho:

Fluxograma1: procedimentos realizados no ambiente SIG

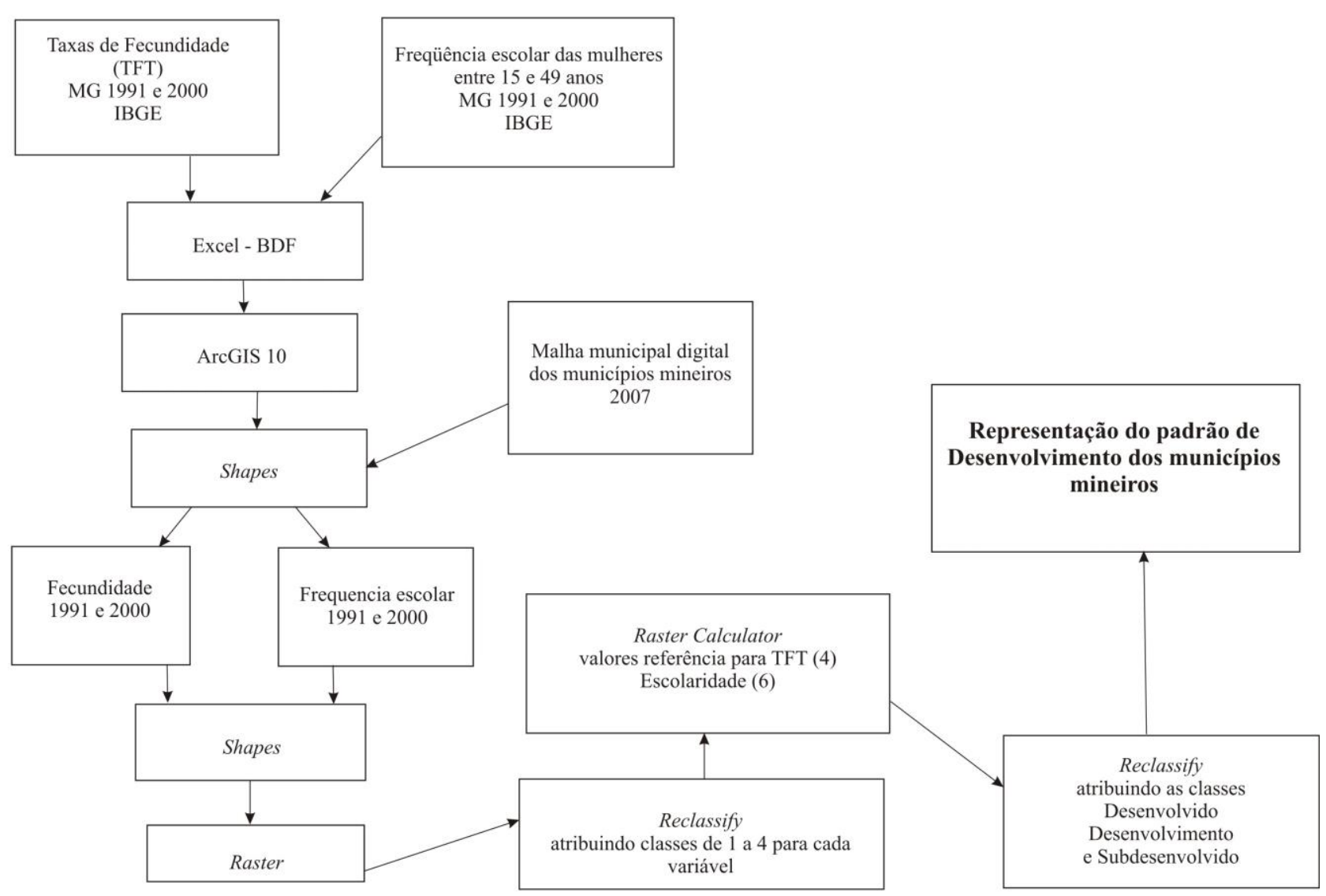




\section{RESULTADOS E DISCUSSÕES}

Os resultados podem ser observados nos mapas temáticos de representação espacial do Padrão de Desenvolvimento dos Municípios Mineiros quanto a Frequência Escolar e a Fecundidade das Mulheres de 15 a 49 anos referentes ao censo de 1991 e 2000 (Figuras 1 e 2).
Muitos demógrafos acreditam que uma maior dedicação aos estudos retarda a iniciação sexual, porque a mulher tem menos tempo livre. Além disso, uma escolaridade elevada proporciona um maior destaque no mercado de trabalho, o que faz com que a mulher pense em ter filhos em idades mais avançadas, quando ela alcança certa estabilidade. Verifica-se que tal padrão não ocorre em 1991 (Figura 1).

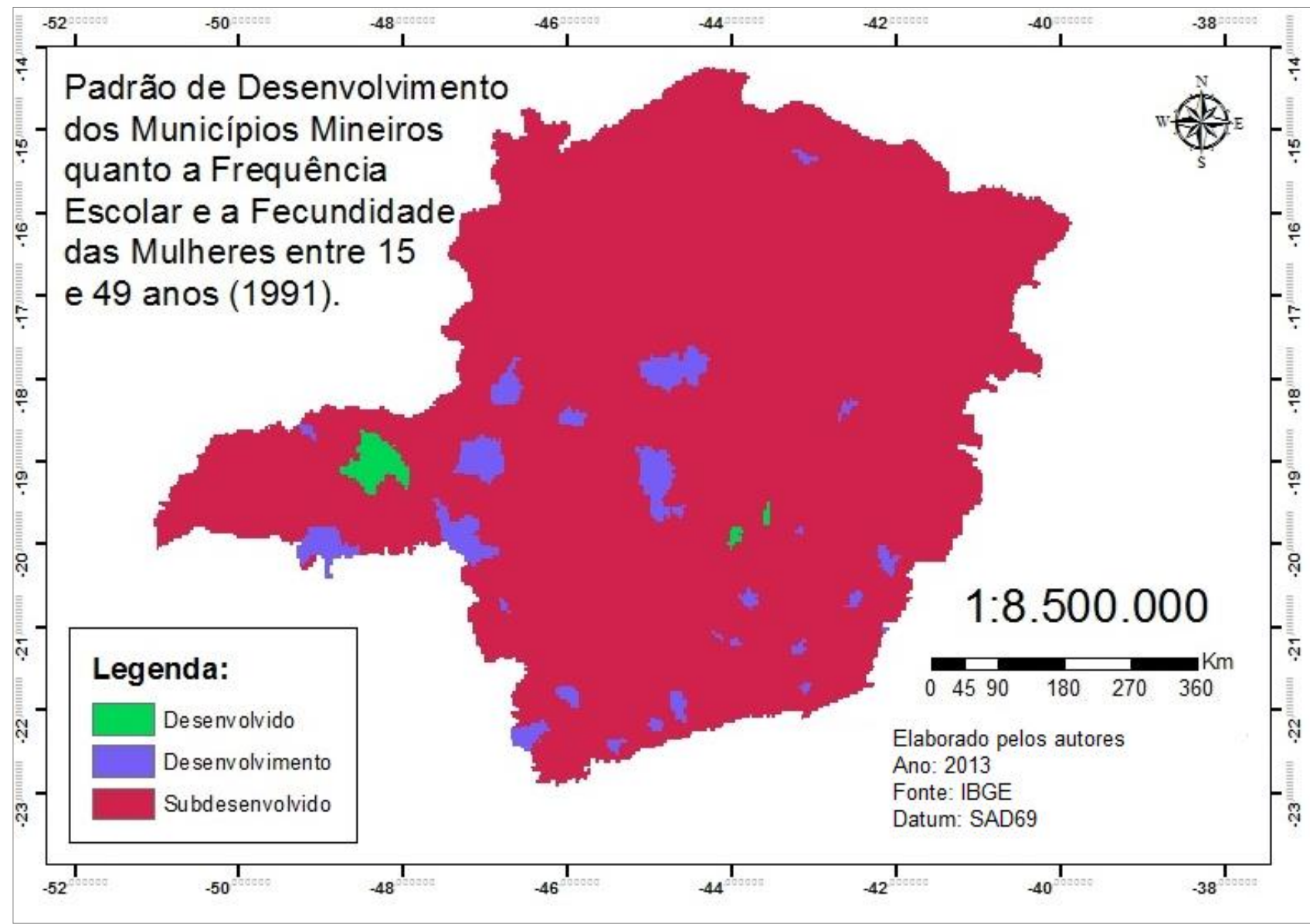

Figura 1 - Mapa de espacialização do Padrão de Desenvolvimento dos Municípios Mineiros quanto a Frequência Escolar e a Fecundidade das Mulheres entre 15 e 49 anos

Percebe-se que na Figura 1, referente ao ano de 1991, a maioria dos municípios do estado apresentava um padrão de desenvolvimento Subdesenvolvido. As taxas de fecundidade ainda eram consideravelmente altas segundo dados do IBGE. A Taxa de
Fecundidade Total entre as mulheres do estado ainda tinha média de 3,2 filhos por mulher. Quanto à frequência escolar no Ensino Médio, a porcentagem de mulheres que estudavam até esse nível ainda era baixa na data analisada. 
Em relação à representação de 2000 mineiros passa a apresentar o padrão (Figura 2), o padrão sofre uma mudança Desenvolvido. significativa. Grande parte dos municípios

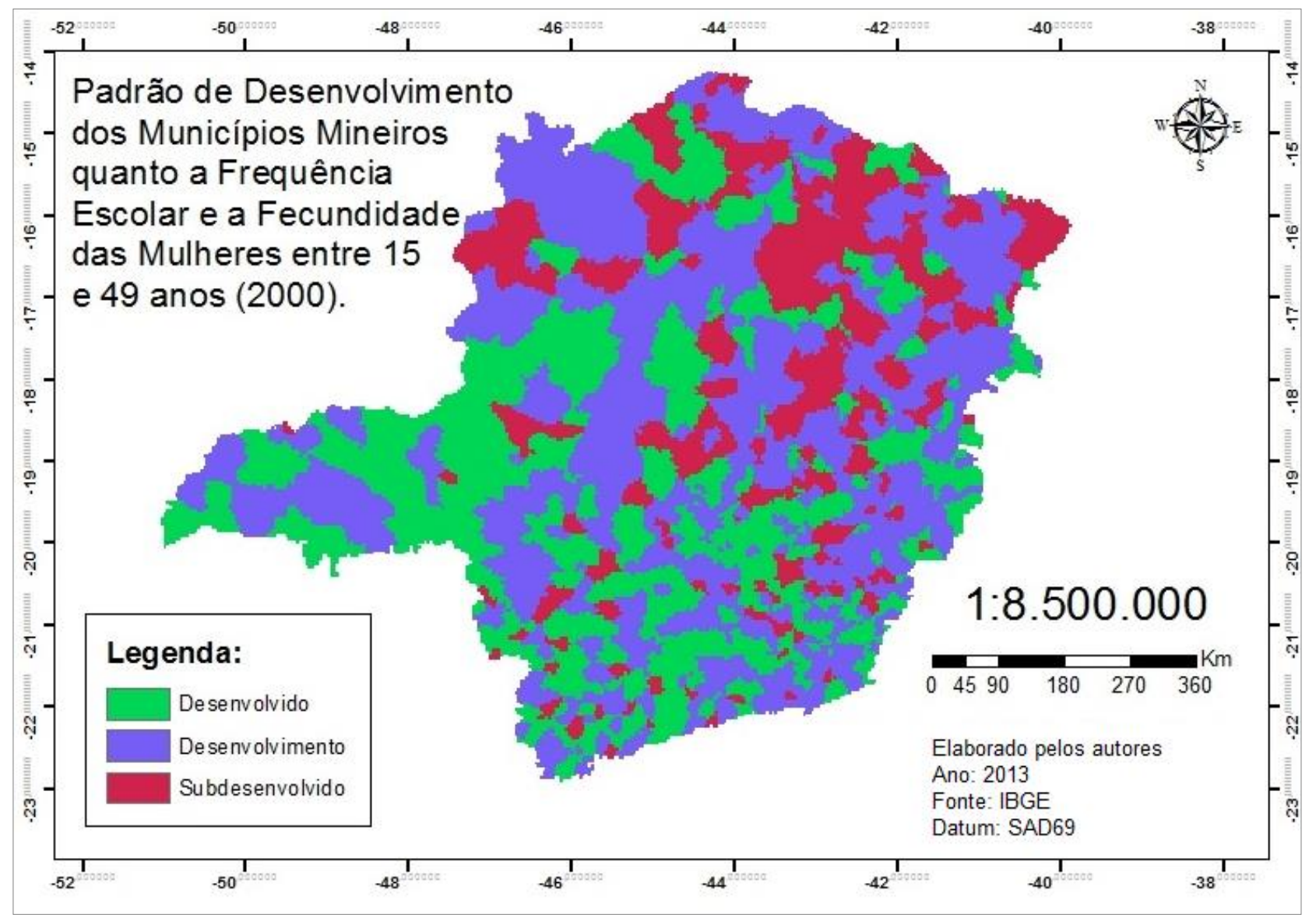

Figura 2 - Mapa de espacialização do Padrão de Desenvolvimento dos Municípios Mineiros quanto a Frequência Escolar e a Fecundidade das Mulheres entre 15 e 49 anos no censo de 2000.

As mesorregiões: Metropolitana de Belo Horizonte, Central Mineira, Oeste e Minas, Campo das Vertentes e Sul/Sudoeste de Minas apresentaram expressiva modificação do Padrão de Desenvolvimento. Em 1991, a maioria dos municípios dessas regiões apresentou padrão de desenvolvimento Subdesenvolvido, já no censo de 2000 o cruzamento das variáveis mostra uma tendência de melhora e uma classificação referente ao padrão Desenvolvimento e Desenvolvido.

Também pode ser aferido que o Triângulo Mineiro pode ser considerado a região mineira com melhor padrão de desenvolvimento, cidades com Uberaba e Uberlândia apresentam índices socioeconômicos elevados em relação às demais. O mapa de 2000 evidencia uma transição rápida no padrão de desenvolvimento proposto. Quase que a totalidade dos municípios ali situados obteve padrão Desenvolvido. Essa modificação pode ser fruto das significativas transformações que sofre a região. O Triângulo Mineiro tem apresentado nas ultimas décadas diversificação produtiva, valores elevados de indicadores sociais e um processo de crescimento urbano 
principalmente nos maiores centros como Uberlândia, Uberaba e Araguari (MARTINS et al.,2007).

A realidade da melhora no Padrão de Desenvolvimento dos municípios mineiro não é representativa em todo o estado. O mapa de 2000 mostra que a maioria nos municípios situados na região do Norte do estado (mesorregiões Norte de Minas. Jequitinhonha e Vale do Mucuri) apresentam índice de classificação Subdesenvolvido. Essa classificação possui respaldo ao aferir que é nessa região que se situam os piores indicadores sociais dentre os municípios mineiros, entretanto, numa analise comparativa entre as duas datas houve, também, melhoria do padrão em vários municípios antes classificados como Subdesenvolvido.

\section{CONSIDERAÇÕES FINAIS}

No Brasil a educação sempre esteve correlacionada com o nível de fecundidade e, apesar da tendência de convergência entre os grupos com acesso a menor e maior disponibilidade de educação, os níveis de fecundidade ainda mantêm um diferencial bastante elevado entre as mulheres pertencentes a categorias extremas desses grupos.

Ao comparar os mapas de classificação do padrão de desenvolvimento dos municípios do estado de Minas Gerais quanto à Frequência Escolar das mulheres e a Fecundidade (dados do IBGE 1991 e 2000 modelados) vemos que o prognóstico de que a crescente população extrapolaria a capacidade de gestão e que acarretaria uma realidade com graves problemas na capacidade de desenvolvimento, moradia, alimentação e políticas públicas tornaram-se de certo modo fracassada, pelo menos, ao associar esses problemas apenas ao crescimento demográfico.

O panorama demográfico modelado pelos resultados apresentados nesse trabalho se mostrou definido nos dois períodos avaliados ao visualizarmos nitidamente em 1991 um nível subdesenvolvido e, em 2000, uma transição estadual para um nível desenvolvido.

Os resultados de evolução corroboram com o fato de que há uma diminuição do crescimento populacional mineiro e que este fator pode estar consorciado a razões como, por exemplo, desenvolvimento de um maior senso de direito no domínio privado, percepção de novas capacidades, aprendizagem de formulação de direitos legais, que são correlacionados diretamente com a realidade de maior acesso à educação.

Atualmente o acesso a maiores níveis de educação, a inserção de mulheres num setor dinâmico da economia e o uso de contraceptivos femininos atinge níveis considerados comparáveis aos dos países desenvolvidos modernos. A redução da fecundidade fez com que as taxas passassem de 4.5 filhos em 1980, 3.5 em 1984 chegando a 2.5 em 1991 (ARILHA et al., 2012).

Mesmo levando em consideração este quadro, o impacto dos nascimentos, num futuro 
breve, afetará diretamente o setor econômico, seja, pelo número de nascimentos, pela manutenção do número de mão-de-obra, crescente número de idosos, ou mesmo, pelo fato das mulheres ainda possuírem a maternidade como projeto de vida tendo cada vez um menor número de filhos.

Diante do grande declínio da taxa de fecundidade no estado, quantificar, localizar e qualificar quais são as áreas em que a fecundidade atinge um nível desenvolvido, além da análise e consequente estudos, favorece a definição de políticas organizacionais de planejamento estratégico no futuro, em consideração, principalmente, do setor econômico e do mercado de trabalho, o que reflete diretamente na manutenção da economia do estado como um todo.

\section{AGRADECIMENTOS}

Os autores agradecem a geógrafa Daniela Oliveira e Cláudio e ao geógrafo Jean Cássio Lima pelas sugestões. Também agradecem ao Prof. Dr. Sergio Donizete Faria pelo auxílio durante a execução do trabalho.

\section{REFERÊNCIAS}

ALVES, J. E. D. A Polêmica Malthus versus Condorcet Reavaliada à Luz da Transição Demográfica. Rio de Janeiro, Escola Nacional de Ciências Estatísticas, v.56, n. 4, 2002.

ARILHA, M.; CAETANO, A. J.; GUEDES, M. C.; MARCONDES, G. S. Diálogos Transversais em Gênero e Fecundidade -
Articulações Contemporâneas. Librum, 2012. v.1. $174 \mathrm{p}$.

BERQUÓ, E.; CAVENAGHI, S. Mapeamento Sócio-econômico e Demográfico dos Regimes de Fecundidade no Brasil e sua Variação entre 1991 e 2000. In: Encontro Nacional de Estudos Populacionais, 14, 2004, Caxambu. Anais... Campinas: ABEP, 2004.

BRITO, F. A transição demográfica no Brasil: as possibilidades e os desafios para a economia e a sociedade. Minas Gerais: UFMG, Cedeplar, 2007 (Textos para a discussão, 318).

FARIA, T. C. A. B.; NOGUEIRA, M.; OLIVEIRA, F. B. Centralidade de Sete Lagoas e sua Relação com os Fluxos Populacionais Desde sua Industrialização Efetiva (1960 2010). In: Encontro Nacional de Estudos Populacionais, 2012, Águas de Lindóia. Anais... Campinas:ABEP, 2012.

IBGE. Censo Demográfico 1991. Disponível em: <http://biblioteca.ibge.gov.br/ /CD1991/CD_1991_resultados\%20preliminares. pdf>.

IBGE. Censo Demográfico 2010. Disponível em: <http://www.censo2010.ibge.gov.br>.

\section{IBGE. Pesquisa Nacional de Amostras a} Domicílio - Síntese dos Indicadores 2009. Disponível em:

<http://www.ibge.gov.br/home/estatistica/popul acao/trabalhoerendimen

to/pnad2009/>.

MARTINS, H. E. P.; JÚNIOR, L. B.; OLIVEIRA, P. L. Urbanização, Migração e Emprego: Uma Análise de Municípios no Triângulo Mineiro e no Sul de Minas. Rev. Pesquisa \& Debate, v.18, n.2(32) p.283-305, 2007.

LANDRY, A. La Révolution Démographique. Etudes et Essais sur les Problèmes de la Population. Paris: Ined. (reedição da publicação de 1934), 1982. 
ORGANIZAÇÃO DAS NAÇÕES UNIDAS. 2011. Relatório sobre a Situação da População Mundial. Nova York: Fundo de População das Nações Unidas, 2011. 132p.

ORGANIZAÇÃO DAS NAÇÔES UNIDAS. 2013. United Nations, Department of Economic and Social Affairs, Population Division (2013). World Population Prospects: The 2012 Revision, Highlights and Advance Tables. Working Paper No. ESA/P/WP.228

YAZAKI, L M. Fecundidade da Mulher Paulista Abaixo do Nível de Reposição, Estudos Avançados, USP, v. 17, n. 49, p.65-86, 2003. 